\title{
Feeling bad about screwing up: emotion regulation and action monitoring in the anterior cingulate cortex
}

\author{
Naho Ichikawa • Greg J. Siegle • Neil P. Jones • \\ Kyoko Kamishima • Wesley K. Thompson • \\ James J. Gross • Hideki Ohira
}

Published online: 18 May 2011

(C) Psychonomic Society, Inc. 2011

\begin{abstract}
This study examined neural features of emotional responses to errors. We specifically examined whether directed emotion regulation of negative emotion associated
\end{abstract}

Electronic supplementary material The online version of this article (doi:10.3758/s13415-011-0028-z) contains supplementary material, which is available to authorized users.

N. Ichikawa $\cdot$ N. P. Jones $\cdot$ W. K. Thompson Department of Psychiatry,

University of Pittsburgh School of Medicine,

Pittsburgh, PA, USA

N. Ichikawa $\cdot$ H. Ohira

Department of Psychology, Nagoya University,

Nagoya, Japan

N. Ichikawa

Department of Psychiatry, Stanford University,

Stanford, CA, USA

\section{J. J. Gross}

Department of Psychology, Stanford University,

Stanford, CA, USA

K. Kamishima

Department of Neuroscience, University of Pittsburgh,

Pittsburgh, PA, USA

W. K. Thompson

Department of Psychiatry, University of California,

La Jolla,

San Diego, CA, USA

G. J. Siegle $(\bowtie)$

Western Psychiatric Institute and Clinic,

3811 O'Hara Street,

Pittsburgh, PA, USA

e-mail: gsiegle@pitt.edu

K. Kamishima

Department of Neuroscience,

Albert Einstein College of Medicine,

New York, NY, USA with error modulates action-monitoring functions of anterior cingulate cortex, including conflict monitoring, error processing, and error prevention. Seventeen healthy adults performed a continuous performance task during assessment by fMRI. In each block, participants were asked either to increase or decrease their negative emotional responses or to react naturally after error commission. Emotion regulation instructions were associated with modulation of rostral and dorsal anterior activity and of their effective connectivity following errors and conflict. Cingulate activity and connectivity predicted subsequent errors. These data may suggest that responses to errors are affected by emotion and that aspects of emotion and cognition are inextricably linked, even during a nominally cognitive task.

Keywords Emotion regulation - Anterior cingulate cortex · Action monitoring · Conflict · Error · fMRI

This study examined whether regulation of negative emotion associated with errors modulates the brain activity associated with self-monitoring of actions. Continuous performance on cognitive tasks is facilitated by an actionmonitoring system that optimizes behavior by monitoring for conflicts, responds to errors, and prevents future errors by signaling the need for greater cognitive control (Botvinick, Cohen, \& Carter, 2004; Botvinick, Nystrom, Fissell, Carter, \& Cohen, 1999; Carter, Braver, Barch, Botvinick, Noll, \& Cohen, 1998). Although action monitoring is often considered to be a cognitive activity devoid of explicit emotional content, features such as conflict and errors have also been associated with emotional reactions (Bush, Luu, \& Posner, 2000; Luu, Tucker, Derryberry, Reed, \& Poulsen, 2003). Initial evidence suggests that negative emotional states modulate activity in the action-monitoring system (Davidson, Lewis, Alloy, Amaral, Bush, Cohen et al., 2002; Gehring, Himle, \& Nisenson, 2000). Thus, our goal was to examine whether 
neural features of action monitoring inherently appear to include components of emotional reactivity and regulation. We pursued this goal by explicitly manipulating emotional contingencies of action monitoring.

Functions of the action-monitoring system, including conflict monitoring, error processing, and error prevention, have specifically been associated with activity in the anterior cingulate cortex (ACC; Barch, Braver, Akbudak, Conturo, Ollinger, \& Snyder, 2001; Botvinick, 2007; Braver, Barch, Gray, Molfese, \& Snyder, 2001; Bush et al., 2000; van Veen \& Carter, 2002; van Veen, Cohen, Botvinick, Stenger, \& Carter, 2001). Greater activity in the dorsal part of the ACC extending into the supplementary motor area (SMA) and the pre-SMA has been associated with conflict monitoring (for a review, see Botvinick et al., 2004) as well as with error processing during nonemotional cognitive tasks (Ullsperger $\&$ von Cramon, 2001). In contrast, greater activity in the ventral part of the ACC, including the rostral ACC (rACC) and subgenual or subcallosal ACC (sACC), has been specifically associated with affective conflict or the detection of emotional distractors during cognitive tasks such as emotion-word Stroop tasks (Mohanty, Engels, Herrington, Heller, Ho, Banich et al., 2007; Ochsner, Hughes, Robertson, Cooper, \& Gabrieli, 2009) and emotional face-word Stroop tasks (Egner, Etkin, Gale, \& Hirsch, 2008; Etkin, Egner, Peraza, Kandel, \& Hirsch, 2006). These results suggest that the ventral ACC may also serve a conflict-monitoring or error-processing function that is specific to emotional distractors, and it further implies that an individual's internal emotional state during a task may also modulate activity within the action-monitoring system. Similarly, the errorrelated negativity (ERN) is an event-related potential (ERP) associated with conflict monitoring and error detection. Higher ERN amplitude has been associated with better performance (for reviews, see Falkenstein, Hoormann, Christ, \& Hohnsbein, 2000; Nieuwenhuis, Holroyd, Mol, \& Coles, 2004). Source localization and designs that combine ERP and fMRI assessments have suggested that the source of the ERN is the rACC (Holmes \& Pizzagalli, 2008; Mathalon, Whitfield, \& Ford, 2003).

Consistent with this hypothesis, negative emotions such as anxiety, worry, and frustration have been observed to modulate the functional activity in the ventral ACC and performance during cognitive tasks, especially for individuals who experience affective disorders such as depression (Johnson, Nolen-Hoeksema, Mitchell, \& Levin, 2009), depression with comorbid anxiety (Engels, Heller, Spielberg, Warren, Sutton, Banich et al., 2010), and obsessive compulsive disorder (Cavanagh, Gründler, Frank, \& Allen, 2010; Ursu, Stenger, Shear, Jones, \& Carter, 2003). Furthermore, patients with rACC lesions displayed impaired posterror slowing, which happens as a behavioral adjustment after error commission for healthy adults (Pellegrino,
Ciaramelli, \& Làdavas, 2007). Yet previous investigations have not examined whether the modulation of ACC actionmonitoring functions is trait related (e.g., associated with a psychopathology) or state related (e.g., associated with a sad mood), or rather reflects inherent responses to emotional stimuli that would occur even in the absence of a specific trait or mood state.

In order to examine relationships of emotional reactivity to action-monitoring functions in healthy adults, we asked participants to up- or down-regulate negative emotions associated with errors during a continuous performance task (CPT; Carter, Macdonald, Botvinick, Ross, Stenger, Noll, \& Cohen, 2000). In the instructions to increase or decrease negative emotions after errors, we did not ask participants to use a specific strategy, because we were interested in the naturalistic regulation strategies that they would usually use after error commission. This could reflect a broad array of strategies, including reappraisal (i.e., cognitive change of the emotional impact of errors) and suppression (i.e., of physical expressions in response to errors).

Finally, the brain mechanisms subserving emotion and cognition are generally analyzed separately, although these systems are well known to interact. Here, we were interested in whether brain responses to emotion regulation instructions were associated not only with activity in specific regions but with modulation of systemwide interactions across the ACC. Anatomical studies have suggested that there are strong connections between dorsal and rostral regions of ACC (Davis, Hutchison, Lozano, Tasker, \& Dostrovsky, 2000; Davis, Taylor, Hutchison, Dostrovsky, McAndrews, Richter et al., 2005). Furthermore, research examining cognitive performance in the presence of emotional load has demonstrated inhibitory associations between rACC and the dorsal ACC/SMA (Bush et al., 2000; Williams, Kemp, Felmingham, Barton, Olivieri, Peduto et al., 2006). The rACC has also been shown to be activated during several studies in which participants were asked to regulate their emotion in response to affective stimuli (Beauregard, Lévesque, \& Bourgouin, 2001; Ochsner, Ray, Cooper, Robertson, Chopra, Gabrieli et al., 2004; Ray, Ochsner, Cooper, Robertson, Gabrieli, \& Gross, 2005). Thus, to understand whether usual inhibitory interactions between the brain mechanisms of emotion and cognitive processing of errors were affected by explicit emotion regulation, we examined modulation of effective connectivity between empirically detected dorsal and ventral action-monitoring regions by regulation instructions using a bivariate functional mixed-effects model. This analysis could add to the existing actionmonitoring and emotion regulation literatures by showing that these domains cannot be considered in isolationrather, emotion regulation would be shown to affect not just the regions associated with each function separately, but 
their systemic interactions as well. We hypothesized that regulation of negative emotion associated with errors would modulate ACC activity. Specifically, we predicted that areas of ventral and dorsal ACC activity would be associated with emotion regulation as well as (a) conflict monitoring, (b) error processing, and (c) error prevention. Whether the effects of emotion regulation and action monitoring were additive (i.e., detectable as a conjunction of activity in response to regulation instructions and action monitoring) or nonlinear (i.e., interaction of regulation condition and action monitoring) was not predicted, and thus both possibilities were examined. In addition, we predicted that there would be modulation of the functional relationships between dorsal and ventral action-monitoring regions associated with both error processing/prevention and emotion regulation. We were specifically interested in the question of whether decreasing negative emotional responses would modulate those functional relationships to cause more error prevention on a trial-by-trial basis. Because the time course of the neural activity associated with error processing was unknown but was estimated to take on the order of seconds, since participants were instructed to continue to regulate for the $10 \mathrm{~s}$ between successive trials, we used a slow-event-related analysis that accounted for arbitrary differences in the shapes of the hemodynamic response to understand differences between the regulation conditions.

Our design had blocks for each explicit emotion regulation condition, as in previous emotion regulation studies. Because performance accuracy is usually high in a CPT (Carter et al., 2000), we used a series of rapid trials punctuated by longer delays following some error and correct trials within blocks. This design allowed for a large number of error trials in a limited time, thereby avoiding the influence of fatigue, but unlike most block designs, also allowed for analysis of the time course of responses to each examined error.

\section{Method}

Participants

The participants included 17 healthy right-handed individuals ( 8 female, 14 Caucasian; ages $20-45$, mean age $=24.2, S D=$ 6.4). The participants reported no health problems and no history of psychiatric problems, as diagnosed via a structured clinical interview (SCID-I; First, Spitzer, Gibbon, \& Williams, 1996). Participants for whom the presence of metallic fragments in their head could not be ruled out by interview were not allowed to participate in the MRI assessment. All participants signed written informed consent, which was approved by the University of Pittsburgh's Institutional Review Board.
Procedure

Before the MRI assessment, participants completed $15 \mathrm{~min}$ of training/calibration on a modified CPT involving four training steps. Then, in the scanner, participants received a fifth training step prior to the scan. Finally, they completed four blocks of the task in different emotion regulation conditions during the scan and answered questions on their emotional and arousal states in the scanner after each block (see the Experimental Session section for details).

\section{Continuous performance task}

Participants were required to judge whether letters presented in the center of a computer screen were Xs $(65 \%)$ or any other letter (35\%) in a modified CPT. Using their right hands, the participants were asked to press the button under their middle finger for $\mathrm{X}$ and the button under their index finger for any other letter. The corresponding keys were counterbalanced across participants. The target stimulus duration was titrated individually in order to obtain a $30 \%$ error rate for each participant, using the following procedure in a training session, which took around $15 \mathrm{~min}$ in total. Following training, participants completed this task under different emotion regulation instructions. The training, the regulation instructions, and finally the task itself, as administered in the scanner, are described below.

\section{Training session}

Phase 1 (for 1 min total) Feedback on performance was presented after each trial. During this training phase, participants were required to respond during the trial (letter+blank screen) in order to avoid a "No Response" message in red letters.

Phase 2 (for 1 and a half minutes total) The stimulus duration was adjusted after every 12 trials on the basis of the number of errors. The interstimulus interval became $30 \mathrm{~ms}$ shorter if there were two or fewer errors, and longer if there were more than two errors.

Phase 3 (for 4 min total) The stimulus duration was again adjusted individually to obtain a $30 \%$ error rate for each participant, by adding $30 \mathrm{~ms}$ for every 6-trial set in which participants got more than two wrong and subtracting $30 \mathrm{~ms}$ for every 6-trial set in which participants got fewer than two wrong. The individually adjusted time range of stimulus presentation was $180-390 \mathrm{~ms}(M=276 \mathrm{~ms}, S D=$ 58) among the participants. This phase mimicked the actual task, as 10-s blank periods were inserted after half of the errors and one sixth of the correct trials. 
Phase 4 (for 4 min total) Participants were asked to do the same task with emotion regulation instructions. They verbally confirmed that they could understand the emotion regulation instructions.

Phase 5 (for 1 min total) During the structural scan before the task, we repeated the first training phase, again with performance feedback, and checked that participants could credibly respond to almost all of the trials with the same speed from the training session in the scanner.

\section{Task instructions for emotion regulation}

Participants were instructed to increase or decrease their emotional responses to errors, or to respond naturally, in different CPT blocks. To help them regulate their emotional responses, participants were given the instructions below, which are based on those from previous emotion regulation studies (Gross, 1998). In the decrease block, the participants were instructed that

We would like you to complete this task as quickly and accurately as possible. To help us understand how people respond cognitively to errors, we would like you to keep to an absolute minimum any emotional responses you may have if you make an error. Remain calm if you make an error. Keep in mind that this is $\mathrm{OK}$, and it happens to everyone in speeded tasks like this. Try to move on quickly and limit any emotional responses you may have.

In the increase block, participants were instructed that

We would like you to complete this task as quickly and accurately as possible. To help us understand how people respond emotionally to errors, we would like you to magnify any emotional responses you may have if you make an error. If you make an error, think of how important it is to avoid errors, and focus on how poorly this reflects on your intelligence. Try to move on quickly but magnify any emotional responses you may have.

In the natural block, the instructions were

We would like you to complete this task as quickly and accurately as possible. To help us understand how people respond to errors, we would like you to respond as naturally as you can if you make an error. Try to move on quickly and neither decrease nor increase any emotional responses you may have. Do not ignore your errors. Just react naturally if you make an error. Do not try to be especially calm or aroused. We want you to react as naturally as possible. Just do it.
Experimental session

Regulation conditions In the scanner, participants were asked to complete four 6-min blocks of the CPT. In order to avoid practice effects, the averaged results of the first and last natural condition blocks were used as a control condition. The emotion regulation conditions, in which participants were required to decrease or increase their emotional responses to errors, were assigned as either the second or the third block, and the order of these conditions was counterbalanced between participants. Before each block, participants were instructed to increase or decrease their emotional responses or to respond naturally. An instruction ("increase response," "decrease responses," or "respond naturally") was present above the target letters throughout each block.

Trials Throughout each 6-min block, single letters were presented for $300 \mathrm{~ms}$, followed by a blank screen for $250 \mathrm{~ms}$. Participants were asked to respond as quickly and accurately as possible. Longer blank periods (10 s) were inserted after every second error trial (whether or not it occurred consecutively with another error trial) and after every seven correct trials, to allow for efficient slow-eventrelated analysis of the fMRI signal, in order to create a mixed intertrial interval (ITI) design (see Fig. 1). During the 10-s blank periods, participants were instructed to keep increasing or decreasing their emotional responses to errors or to maintain a natural response to errors while looking only at the instruction sentence described above for each regulation condition. We used only the 10 -s blank periods for fMRI data analysis; the other, short-ITI trials were not used for the fMRI data analyses described below.

After each block, participants answered questions about their emotional state and arousal level, which were presented on the monitor in the scanner and answered with the response buttons. In particular, we asked participants to rate their degree of experienced negative emotion after error and correct trials separately, in order to make sure that the participants understood the emotion regulation instructions and tried to regulate negative emotional valence during each block condition.

\section{fMRI data handling}

\section{fMRI data acquisition}

The fMRI data were collected using the 3T GE Signa MRI scanner in the MR Center of Presbyterian Hospital in Pittsburgh. Stimuli were presented in the scanner using a video projection device, projecting images onto a backprojection screen at the participant's chest that was viewed in a mirror attached to the head coil. Stimulus presentation 


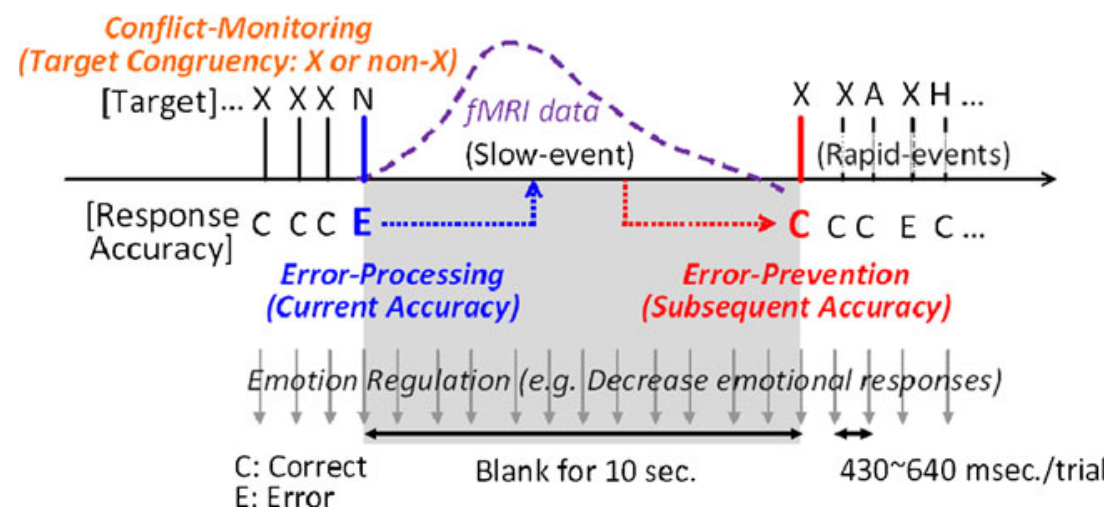

Fig. 1 Experimental protocol: a continuous performance task employed a mixed intertrial interval design (c: Correct response; e: Error response). The fMRI data during slow events (followed by a blank screen for $10 \mathrm{~s}$ ) were analyzed based on current and subsequent

and registration of responses were controlled by a Microsoft Windows-based computer running E-Prime (Psychology Software Tools, Inc.). Participants' button press responses were recorded using an radio-frequency-shielded response box and cable connected to the computer (Psychology Software Tools glove). Thirty axial slices $(3.125 \times 3.125 \times$ $3.2 \mathrm{~mm}$ voxels) per trial were acquired perpendicular to the AC-PC line using a $\mathrm{T}^{*}$ weighted reverse-spiral pulse sequence $(\mathrm{TR}=1,500 \mathrm{~ms}, \mathrm{TE}=26 \mathrm{~ms}, \mathrm{FOV}=20 \mathrm{~cm}$, flip= 60 ). This sequence allowed 30 slices to be acquired every $1.5 \mathrm{~s}$. Scanning began with stimulus onset. In previous studies, this technique was sufficient to allow for examination of the temporal dynamics of activation in multiple task conditions. Anatomical scans were acquired at the same locations as the functional imaging scans, using a standard T1-weighted pulse sequence $(\mathrm{TR}=350 \mathrm{~ms}, \mathrm{FOV}=20 \mathrm{~cm}, 0.78125 \times 0.78125 \times$ $3.2 \mathrm{~mm}$ voxels, 34 slices).

\section{fMRI data preprocessing}

AFNI (Cox, 1996), AIR ("automated image registration"; Woods, Cherry, \& Mazziotta, 1992), NeuroImaging Software (NIS; http://kraepelin.wpic.pitt.edu/nis/), and the Java Graphical Computing Environment for Neuroimaging Analysis (Fissell et al., 2003) were applied for analysis of the fMRI imaging data. Functional images were reconstructed from K-space using NIS. As preprocessing steps, we used the AFNI-3dTshift function for slice-time correction, AFNI-3dvolreg for motion correction, NIS-nismaps for making statistical map images, AFNI-3dTsatat for making a median map image, NIS-niscorrect for removing linear trend, AFNI-3dcalc for normalizing time series data sets to percent change values, and NIS-nisfilter for temporal smoothing (5point middle-peaked filter). These functional images were coregistered to individual resliced structural T1-weighted images with a rigid transform, then co-registered to individual performance accuracy. Action-monitoring functions were analyzed based on conflict monitoring (target: $\mathrm{X}$ or non-X), error processing (current performance accuracy), and error prevention (subsequent performance accuracy)

high-resolution images (SPGR; $0.9375 \times 0.9375 \times 1.5$ voxels) via a rigid transform, and finally co-registered to a standard reference brain (Montreal Neurologic Institute: MNI Colin27) via a 32-parameter nonlinear warping procedure with AIR and spatially smoothed with a 6-mm full-width-at-halfmaximum (FWHM) three-dimensional Gaussian filter. A single region of interest (ROI) encompassing the dorsal, rostral, and subgenual ACC was specified using the AFNI Talairach Atlas with Anterior Cingulate constrained to $y<48$ and Cingulate Gyrus constrained to $y>-9$, in light of previous studies (Bush, Shin, Holmes, Rosen, \& Vogt, 2003; McCormick et al., 2006).

\section{fMRI data analysis}

The primary analyses involved three-way, repeated measures voxel-wise ANOVAs in which we examined the effects of emotion regulation (decrease, natural, increase) and action monitoring, including conflict monitoring (target congruency: $\mathrm{X}$, non-X), error processing (current accuracy: correct, error), and error prevention (subsequent accuracy: correct, error), on the trial-related BOLD signal using Nisanova. Trials with 10 -s blank periods in the mixed-ITI design were used for the fMRI data analysis (1) in order to allow for assumption-free analysis of the time course of the hemodynamic response to errors and (2) so that the titrated stimulus presentation rate for each individual did not affect the fMRI data during the 10-s blank.

In each analysis, Participants was a random factor, and Emotion Regulation Condition, Action Monitoring, and Scan Within a Trial (there were seven 1.5 -s scans per 10-s trial) were fixed factors. We analyzed effects in which an interaction with scan was present because this indicates an effect of condition on the shape of the hemodynamic response. Only correct trials were considered for the analysis of conflict-monitoring effects in order to disentangle the 
effects of error and conflict. Pretask titration yielded sufficient proportions of correct and error trials for analyses $(\geq 10$ per condition); the numbers of trials that contributed to the percent signal change estimates analyzed in each condition are shown in aggregate in Table S2 in the supplementary materials, and those for each participant in relation to the fMRI percent signal change are in supplementary Figure S5. Table S3 shows that the participants with the fewest trials did not drive the basic character of the results.

Tests of interactive effects of emotion regulation and action monitoring

To test our a priori hypothesis that emotion regulation interacts with action monitoring, and specifically with ACC functioning, we examined the following models of threeway repeated measures ANOVAs for the ACC ROI: (a) Target Congruency $\times$ Emotion Regulation $\times$ Scan Interaction (conflict monitoring), (b) Current Accuracy $\times$ Emotion Regulation $\times$ Scan Interaction (error processing), and (c) Subsequent Accuracy $\times$ Emotion Regulation $\times$ Scan Interaction (error prevention). Secondary whole-brain analyses were performed to consider the entire brain volume. Statistical maps were thresholded at $p<0.005$ with a contiguity threshold empirically determined via AFNI's AlphaSim, based on the spatial autocorrelation of the maps in the ROI analysis using the ACC mask, to test our a priori hypothesis in the context of conflict monitoring (Target Congruency $\times$ Emotion Regulation $\times$ Scan: 5 voxels), error processing (Current Accuracy $\times$ Emotion Regulation $\times$ Scan: 5 voxels), and error prevention (Subsequent Accuracy $\times$ Emotion Regulation $\times$ Scan: 6 voxels $)$. Similarly in the whole-brain analysis of the following three-way repeated measures ANOVAs, statistical maps were thresholded at $p<$ 0.005 for (a) conflict monitoring (Target Congruency $\times$ Emotion Regulation $\times$ Scan, 23 voxels; Target Congruency $\times$ Scan, 23 voxels; Emotion Regulation $\times$ Scan, 26 voxels), (b) error processing (Current Accuracy $\times$ Emotion Regulation $\times$ Scan, 25 voxels; Current Accuracy $\times$ Scan, 57 voxels; Emotion Regulation $\times$ Scan, 25 voxels), and (c) error prevention (Subsequent Accuracy $\times$ Emotion Regulation $\times$ Scan, 21 voxels; Subsequent Accuracy $\times$ Scan, 29 voxels; Emotion Regulation $\times$ Scan, 26 voxels).

Testing additive effects of emotion regulation and conflict monitoring

To test the hypothesis that emotion regulation adds to the effects of conflict monitoring, error processing, and error prevention in specific regions, we did conjunction analyses to find regions that were characterized by effects of both emotion regulation and action monitoring using two-way repeated measures ANOVAs [i.e., the following specific interactions: (a) Target Congruency $\times$ Scan $(p<0.05)$ and Emotion Regulation $\times$ Scan $(p<0.05)$; (b) Current Accuracy $\times$ Scan $(p<0.05)$ and Emotion Regulation $\times$ Scan $(p<0.05)$ interactions; or (c) Subsequent Accuracy $\times$ Scan and Emotion Regulation $\times$ Scan $(p<0.05)]$. Thus, since conjunction maps multiplied the independent probabilities of $p<0.05$, each was examined at an effective significance of $p<0.0025$. An empirically determined contiguity threshold was 17 voxels for target congruency, 28 voxels for current accuracy, and 19 voxels for subsequent accuracy. These contiguous voxel thresholds were imposed on each of the component maps to preserve the analysis-wise error rate at $p<0.05$. Huyn-Feldt corrections were applied to these repeated measures ANOVA results to correct for possible violations of sphericity. Scan effects were included in these ANOVA analyses to allow for differences in the time course of activity among conditions associated with instructed prolonged regulation during the 10-s ITI.

\section{Results}

The following analyses were used to better understand the neural mechanisms of emotion regulation in response to errors. First, to assure that emotion regulation was successful at a subjective level, the self-reported success and emotion for each block were compared. Then, to be sure that the neural phenomena we examined reflected behavioral mechanisms, differences in response times (RTs) to error and subsequenterror trials were examined. To examine effects of the regulatory instructions on the shape of the hemodynamic response as a function, specifically, of action monitoring, we report the results of ANOVAs examining Regulation Condition $\times$ Error or Conflict $\times$ Time (scan-within-a-trial). That said, it was possible that the observed neural activity might be highest for regulation of action monitoring, but because of additive (independent) rather than multiplicative (i.e., interacting) effects of action monitoring and regulation. To test this alternate formulation, we examined conjunction analyses for the action-monitoring and regulation effects. Finally, to combine our behavioral and $\mathrm{AMRI}$ data in order to understand the function effects of regulatory control, we used a bivariate functional mixed-effects model to estimate the directional neural contributions of regions associated with action monitoring and regulation on each other and on the subsequent trial's RTs. This model helped to answer to our main question of how explicit emotion regulation modulates the systemic functioning of error-related dorsal and ventral regions.

Manipulation checks for emotion regulation

As manipulation checks, we examined whether selfperceived success, arousal, and negative emotion varied 
with regulation condition (decreased, natural, increase) via one-way repeated measures ANOVAs. The ratings of negative emotion after error trials significantly varied with regulation of emotional reactions $[F(2,32)=7.14, p<0.005$, $\left.\eta_{\mathrm{p}}{ }^{2}=0.31\right]$, with the linear trend that the participants reported less negative emotion in the decrease condition $(M=2.76, S D=0.16)$ and more negative emotion in the increase condition $(M=3.94, S D=0.23)$ than in the natural condition $(M=3.44, S D=0.15)$ after errors (i.e., decrease $<$ natural $<$ increase; linear trend $t=3.90, p<0.001, R^{2}=0.49$ ). Negative affect was higher for error than for correct trials (see Figs. S1 and S2 in the supplementary materials). Ratings of general arousal state varied with emotion regulation $\left[F(2,32)=5.49, p<0.01, \eta_{\mathrm{p}}{ }^{2}=0.26\right]$; they were lower in the decrease condition $(M=2.41, S D=0.24)$ than in either the increase $(M=3.35, S D=0.30 ; t=2.70, p<0.05$, Cohen's $d=0.96]$ or the natural condition $(M=2.94, S D=$ $0.17 ; t=2.67, p<0.05, d=0.94)$, which did not differ ( $t=$ $1.46, p=0.16, d=0.52)$. Self-perceived success at regulating emotion varied significantly with regulation condition $[F(2$, $\left.32)=3.43, p<0.05, \eta_{\mathrm{p}}{ }^{2}=0.18\right]$; it was lower for the decreasing $(M=3.41, S D=0.24)$ than for the increasing $(M=$ 4.12, $S D=0.15)$ condition $(t=2.14, p<0.05, d=0.76)$. There was no significant difference between the increase and natural conditions ( $t=0.86, p=0.40, d=0.30$ ) for self-perceived success. The actual strategy used by each participant was reported in a debriefing session so that we could understand how they followed our instructions (see the Results of Free Descriptions in Table S1 and Figure S3 in the supplementary materials). These results show that the emotion regulation instructions successfully manipulated subjective ratings on negative emotion after errors.
We also examined order effects for the emotion regulation conditions by comparing the group who completed the decrease condition before the increase condition $(n=8)$ to those who completed the increase condition before the decrease condition $(n=9)$. There was no significant order effect for subjective ratings on negative emotion after error trials [decrease, $t(15)=-0.31, p=0.76$; increase, $t(15)=-0.42$, $p=0.68$ ], arousal level [decrease, $t(15)=0.82, p=0.43$; increase, $t(15)=1.77, p=0.10]$, and successfulness of emotion regulation [decrease, $t(15)=1.34, p=0.20$; increase, $t(15)=$ $0.85, p=0.41]$.

\section{Behavioral data}

Table 1 displays the mean accuracy and RTs in each condition. The reported error rates were generally below $30 \%$ because (1) some responses were over the time limit, and thus were counted as nonresponses rather than errors, and (2) some people performed better in the experimental sessions than in the practice sessions $(n=9$ for the decrease, $n=8$ for the increase, and $n=11$ for the natural condition). That said, this study surpassed a primary hurdle of errorrelated studies, in that the obtained error response rates were sufficient for fMRI analysis due to our idiosyncratic titration procedures. As reported in previous studies (e.g., Mathalon et al., 2003), the congruency of the target stimulus expectation caused by frequency influenced task performance. However, neither the interaction effect of emotion regulation with target congruency $[F(2,32)=1.25$, $\left.p=0.30, \eta_{\mathrm{p}}{ }^{2}=0.07\right]$ nor the main effect of emotion regulation $\left[F(2,32)=1.89, p=0.17, \eta_{\mathrm{p}}{ }^{2}=0.11\right]$ was significant on performance accuracy (i.e., correct response rate). There

Table 1 Mean accuracy and response times for each emotion regulation condition

\begin{tabular}{|c|c|c|c|c|c|c|}
\hline \multirow[b]{2}{*}{ Conditions $^{\mathrm{a}}$} & \multicolumn{3}{|l|}{ Rates $(\%)$} & \multicolumn{3}{|c|}{ Response Time (ms) } \\
\hline & Total $[M(S E)]$ & Congruent & Incongruent & Total $[M(S E)]$ & Congruent & Incongruent \\
\hline \multicolumn{7}{|c|}{ Natural condition } \\
\hline Correct & $72.5(3.2)$ & $77.3(2.8)$ & $65.4(4.1)$ & $372(9)$ & $361(9)$ & $391(9)$ \\
\hline Error & $12.7(2.2)$ & $10.3(1.9)$ & $16.2(2.8)$ & $334(11)$ & $341(11)$ & $326(11)$ \\
\hline Nonresponse & $14.8(2.7)$ & $12.4(2.6)$ & $18.4(3.0)$ & & & \\
\hline \multicolumn{7}{|c|}{ Decrease condition } \\
\hline Correct & $68.6(4.0)$ & $72.5(3.6)$ & $62.7(4.6)$ & $370(10)$ & $355(9)$ & $395(11)$ \\
\hline Error & $14.2(2.8)$ & $12.6(2.7)$ & $16.6(3.1)$ & $323(14)$ & $325(16)$ & $321(13)$ \\
\hline Nonresponse & $17.2(3.4)$ & $14.9(3.1)$ & $20.8(4.0)$ & & & \\
\hline \multicolumn{7}{|c|}{ Increase condition } \\
\hline Correct & $70.6(2.9)$ & $75.9(2.5)$ & $62.6(3.9)$ & $368(10)$ & $356(10)$ & $389(12)$ \\
\hline Error & $14.1(2.1)$ & $11.2(2.0)$ & $18.5(2.7)$ & $329(9)$ & $353(15)$ & $314(8)$ \\
\hline Nonresponse & $15.3(2.2)$ & $12.9(2.2)$ & $18.8(2.6)$ & & & \\
\hline
\end{tabular}

${ }^{\mathrm{a}}$ Responses recorded before and after the response limit were regarded as non-responses. Averages of the response rates and response times are shown in the table, and standard errors of the means are shown in parentheses 
was a main effect of target congruency on performance accuracy $\left[F(1,16)=37.18, p<0.001, \eta_{\mathrm{p}}{ }^{2}=0.70\right]$. In the congruent trials, in which the congruent target stimulus $\mathrm{X}$ was presented, accuracy was higher than in the incongruent trials, in which any letter but $\mathrm{X}$ was presented. There were no condition-related differences in nonresponse rates $[F(2$, $\left.32)=1.19, p=0.32, \eta_{\mathrm{p}}{ }^{2}=0.07\right]$. In subsequent analyses, nonresponses were excluded from the analysis. RTs were influenced by performance accuracy and the congruency of the target expectation, but not by emotion regulation. The interaction of target congruency and response accuracy (correct or error) for RTs was significant $[F(1,15)=30.25$, $\left.p<0.001, \eta_{\mathrm{p}}{ }^{2}=0.67\right]$, though neither of the interactions of emotion regulation with stimulus congruency $[F(1.52$, $\left.22.8)=1.92, p=0.18, \eta_{\mathrm{p}}{ }^{2}=0.11\right]$ or with response accuracy $\left[F(1.58,23.7)=0.26, p=0.73, \eta_{\mathrm{p}}{ }^{2}=0.02\right]$ was significant.

There was an RT and performance accuracy trade-off observed in all of the emotion regulation conditions. Longer RTs were associated with higher accuracy (overall $r=0.61, S E=0.05)$. The correlation between RT and accuracy was not significantly different among emotion regulation conditions $[F(2,32)=0.08, p=0.93]$ in one-way repeated ANOVA, and there was no significant order effect between the groups who completed the decrease before the increase condition, or vice versa, in a mixed-model Task Order $\times$ Emotion Regulation Condition ANOVA on either performance accuracy $[F(2,28)=0.40, p=0.68]$ or RTs for correct trials $[F(2,28)=1.73, p=0.20]$.

For behavioral adjustments, we examined the difference between posterror and postcorrect performance accuracy and RTs after the slow trials with 10-s ITIs. The posterror accuracy did not show any significant difference among regulation conditions in a one-way repeated ANOVA $[F(2,32)=0.53$, $p=0.59]$, and there was no task order effect in a mixedmodel ANOVA (Task Order $\times$ Emotion Regulation, $F(2,28)=$ $0.25, p=0.78$; task order, $F(1,14)=0.68, p=0.42]$. Posterror slowing also showed neither significant differences among regulation conditions in a one-way repeated ANOVA $[F(2,32)=0.47, p=0.63]$ nor an interaction of emotion regulation and task order $[F(2,28)=0.17, p=0.85]$. However, the main effect of task order was significant for posterror slowing $[F(1,14)=6.50, p<0.05]$, showing that the group who completed the decrease condition before the increase condition showed posterror slowing $(M=16.3, S E=12.3)$, whereas the other group, who completed the increase condition before the decrease condition, did not $(M=-42.8, S E=-23.0)$.

Although we titrated error rates for each participant in the training session, error rates showed a positive correlation with self-perceived negative emotion after errors in the natural conditions $(r=0.51, p<0.05)$, but not in the decrease $(r=-0.20, p=0.44)$ or the increase $(r=0.39, p=0.13)$ conditions. That is, having a negative emotion after errors was not associated with task performance during explicit emotion regulation by the instructions, although such a correlation was significant in the natural condition.

\section{Functional imaging data}

One participant each in the analyses of conflict monitoring and error prevention was excluded because of an insufficient number of trials for analyses, based on their performance (i.e., $n=16$ for conflict monitoring and error prevention, $n=17$ for error processing). Tables 2 and 3 show significant regions for each test, with the centroids of clusters listed in Talairach coordinates (Talairach \& Tournoux, 1988). In all figures, hemodynamic responses are displayed as the average change
Table 2 ROI analysis for the anterior cingulate cortex (ACC): Regions and centroid locations showing significant three-way interaction effects $(p<0.005)$ or two-way conjunction effects $(p<0.0025)$

\begin{tabular}{|c|c|c|c|c|c|c|c|}
\hline Side & Region & Brodmann's Area & Volume $\left(\mathrm{mm}^{3}\right)$ & $X$ & $Y$ & $Z$ & $F$ value \\
\hline \multicolumn{8}{|c|}{ Conflict Monitoring } \\
\hline \multicolumn{8}{|c|}{ Conjunction effect: (Target Congruency $\times$ Scan and Emotion Regulation $\times$ Scan) } \\
\hline \multicolumn{8}{|c|}{ Interaction effect: $($ Target Congruency $\times$ Emotion Regulation $\times$ Scan $)$} \\
\hline $\mathrm{L} / \mathrm{R}$ & Subgenual ACC & BA 25 & 156 & 0 & 11 & -4 & 2.86 \\
\hline \multicolumn{8}{|c|}{ Error Processing } \\
\hline \multicolumn{8}{|c|}{ Conjunction effect: (Current Accuracy $\times$ Scan and Emotion Regulation $\times$ Scan) } \\
\hline $\mathrm{R}$ & Dorsal ACC & BA 24 & 281 & 2 & 10 & 34 & 3.27 \\
\hline $\mathrm{R}$ & Rostral ACC & BA 32 & 281 & 12 & 44 & 6 & 3.27 \\
\hline \multicolumn{7}{|c|}{ Interaction effect: (Current Accuracy $\times$ Emotion Regulation $\times$ Scan) } & \\
\hline \multicolumn{8}{|c|}{ Error Prevention } \\
\hline \multicolumn{8}{|c|}{ Conjunction effect: (Subsequent Accuracy $\times$ Scan and Emotion Regulation $\times$ Scan) } \\
\hline $\mathrm{R}$ & Rostral ACC & BA 24 & 594 & 3 & 41 & 10 & 3.82 \\
\hline \multicolumn{8}{|c|}{ Interaction effect: $($ Subsequent Accuracy $\times$ Emotion Regulation $\times$ Scan $)$} \\
\hline $\mathrm{R}$ & Subcallosal ACC & BA $24 / 32$ & 250 & 9 & 35 & 0 & 3.16 \\
\hline
\end{tabular}


Table 3 Whole-brain analysis: Regions and centroid locations showing significant three-way interaction effects $(p<0.005)$ or two-way conjunction effects $(p<0.0025)$

\begin{tabular}{|c|c|c|c|c|c|c|c|}
\hline Side & Region & Brodmann's Area & Volume $\left(\mathrm{mm}^{3}\right)$ & $X$ & Y & $Z$ & $F$ value \\
\hline \multicolumn{8}{|c|}{ Conflict Monitoring } \\
\hline \multicolumn{8}{|c|}{ Conjunction effect: (Target Congruency $\times$ Scan and Emotion Regulation $\times$ Scan) } \\
\hline \multicolumn{8}{|c|}{ Interaction effect: $($ Target Congruency $\times$ Emotion Regulation $\times$ Scan $)$} \\
\hline \multicolumn{8}{|c|}{ Error Processing } \\
\hline \multicolumn{8}{|c|}{ Conjunction effect: (Current Accuracy $\times$ Scan and Emotion Regulation $\times$ Scan) } \\
\hline \multicolumn{8}{|c|}{ Error $>$ Correct and Increase $>$ Decrease } \\
\hline $\mathrm{L} / \mathrm{R}$ & Supplementary Motor Area & BA 6 & 531 & 0 & -5 & 58 & 8.57 \\
\hline $\mathrm{R}$ & Superior Frontal Gyrus & BA 6 & 563 & 3 & 6 & 58 & 6.01 \\
\hline $\mathrm{L}$ & Precuneus & BA $7 / 19$ & 625 & -24 & -76 & 45 & 5.06 \\
\hline $\mathrm{R}$ & Precentral Gyrus & BA 44 & 1,063 & 52 & 6 & 6 & 8.04 \\
\hline $\mathrm{R}$ & Insula & BA 13 & 688 & 48 & 3 & 5 & 8.04 \\
\hline $\mathrm{R}$ & Superior Temporal Gyrus & BA 22 & 2,438 & 54 & 3 & 5 & 7.42 \\
\hline $\mathrm{L}$ & Inferior Frontal Gyrus & BA 45 & 1,250 & -54 & 17 & 5 & 12.81 \\
\hline $\mathrm{R}$ & Inferior Frontal Gyrus & BA 45 & 875 & 54 & 14 & 0 & 5.15 \\
\hline \multicolumn{8}{|c|}{ Interaction effect: $($ Current Accuracy $\times$ Emotion Regulation $\times$ Scan $)$} \\
\hline \multicolumn{8}{|c|}{ Error : Increase $>$ Natural, Decrease } \\
\hline $\mathrm{L} / \mathrm{R}$ & Medial Frontal Gyrus & BA 10 & 688 & 0 & 59 & 26 & 4.71 \\
\hline $\mathrm{L}$ & Medial Frontal Gyrus & BA $9 / 10$ & 156 & -3 & 59 & 29 & 3.53 \\
\hline $\mathrm{R}$ & Precentral Gyrus & BA 6 & 563 & 45 & -15 & 34 & 2.9 \\
\hline $\mathrm{R}$ & Postcentral Gyrus & BA 4 & 188 & 39 & -18 & 40 & 2.9 \\
\hline $\mathrm{R}$ & Postcentral Gyrus & BA 2 & 875 & 48 & -18 & 26 & 3.91 \\
\hline \multicolumn{8}{|c|}{ Error : Increase, Natural $>$ Decrease } \\
\hline $\mathrm{R}$ & Middle Frontal Gyrus & BA 6 & 1,656 & 33 & 11 & 50 & 3.93 \\
\hline $\mathrm{R}$ & Middle Frontal Gyrus & BA 8 & 94 & 36 & 14 & 48 & 3.33 \\
\hline \multicolumn{8}{|c|}{ Error Prevention } \\
\hline \multicolumn{8}{|c|}{ Conjunction effect: (Subsequent Accuracy $\times$ Scan and Emotion Regulation $\times$ Scan) } \\
\hline \multicolumn{8}{|c|}{ Error $<$ Correct and Increase $>$ Decrease } \\
\hline $\mathrm{L} / \mathrm{R}$ & Anterior Cingulate (Rostral) & BA 32 & 594 & 3 & 41 & 10 & 3.27 \\
\hline $\mathrm{R}$ & Medial Frontal Gyrus & BA 10 & 656 & 11 & 50 & 11 & 3.27 \\
\hline \multicolumn{8}{|c|}{ Interaction effect: (Subsequent Accuracy $\times$ Emotion Regulation $\times$ Scan) } \\
\hline \multicolumn{8}{|c|}{ Error : Increase $>$ Decrease } \\
\hline $\mathrm{R}$ & Putamen & $\mathrm{N} / \mathrm{A}$ & 438 & 27 & -3 & 2 & 3.22 \\
\hline
\end{tabular}

from the first scan of long-delay trials. Initial analyses were restricted to the $\mathrm{ACC}$, on the basis of our a priori hypothesis, and were followed with whole-brain analyses to examine the extent to which a broader network was characterized by the same processes.

\section{ACC ROI analysis}

Interaction analyses Whether the emotion regulation would show nonlinear effects on action-monitoring processes in the ACC was examined via three-way repeated measures ANOVAs. Only the subgenual/subcallosal clusters showed nonlinear effects of emotion regulation, as described below.

Conflict monitoring (target congruency) A significant three-way interaction effect of Conflict Monitoring $\times$ Emotion Regulation $\times$ Scan was found in a cluster within the sACC (BA25; centroid: $x=0, y=11, z=-4 ; F(7.5$, $111.7)=3.18, p<0.005, \eta_{\mathrm{p}}{ }^{2}=0.18$; see Fig. 2, top]. There were significant differences among emotion regulation conditions after the congruent target stimuli $[F(7.5,111.8)=$ 2.41, $\left.p<0.05, \eta_{\mathrm{p}}{ }^{2}=0.14\right]$. The sACC showed higher activation in the increase condition $(t=2.83, p<0.05, d=$ 
Conflict Monitoring (target congruency $\mathrm{x}$ emotion regulation $\mathrm{x}$ scan)

Subgenual ACC [centroid: $\mathrm{x}=0, \mathrm{y}=11, \mathrm{z}=-4$ ]

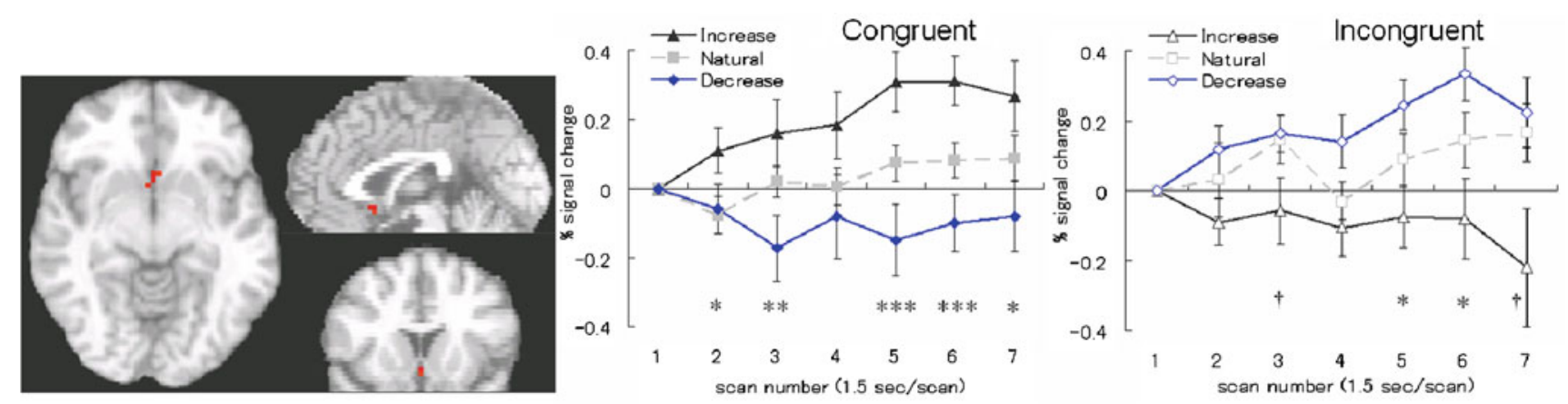

Error Prevention (subsequent accuracy x emotion regulation x scan)

Subcallosal ACC [centroid: $\mathrm{x}=9, \mathrm{y}=35, \mathrm{z}=0$ ]
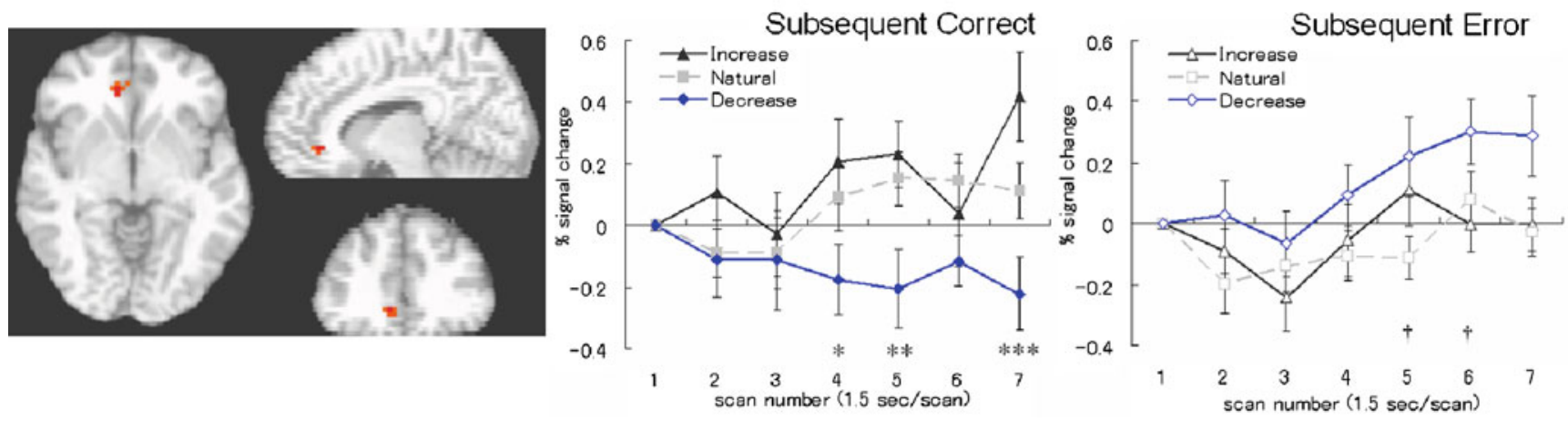

Fig. 2 Interaction effects of emotion regulation and conflict monitoring or error prevention in the ACC. Top: Subgenual ACC cluster and the time course of its functional activity (conflict monitoring: Current
Accuracy $\times$ Emotion Regulation $\times$ Scan). Bottom: Subcallosal ACC cluster and its time course (error prevention: Subsequent Accuracy $\times$ Emotion Regulation $\times$ Scan)
1.03) and lower activation in the decrease condition $(t=$ 2.34, $p<0.05, d=0.86)$, as compared to the natural condition, at Scan 6. However, this pattern was not observed with the incongruent target stimuli $(F(4.5,67.2)=$ $\left.1.49, p=0.21, \eta_{\mathrm{p}}{ }^{2}=0.09\right]$.

Error processing (current accuracy) No significant threeway Current Accuracy $\times$ Emotion Regulation $\times$ Scan interactions were observed in the ACC.

Error prevention (subsequent accuracy) The three-way Subsequent Accuracy $\times$ Emotion Regulation $\times$ Scan interaction was significant in a cluster within the sACC (BA24/32; centroid: $x=9, y=35, z=0 ; F(12,180)=2.99, p<0.001, \eta_{\mathrm{p}}{ }^{2}=$ 0.17; see Fig. 2, bottom]. There were significant differences among emotion regulation conditions before subsequent correct trials $\left[F(5.9,88.8)=3.01, p<0.01, \eta_{\mathrm{p}}{ }^{2}=0.17\right]$. The $\mathrm{SACC}$ region displayed higher activation in the increase than in the natural condition at Scan $7[t(16)=2.25, p<0.05, d=$ $0.82]$, both of which showed higher activation than the decrease condition $[t(16)=2.85, p<0.05, d=1.04]$. However, there was no significant difference associated with emotion regulation before subsequent error trials in this region $\left[F(8.0,119.9)=1.67, p=0.11, \eta_{\mathrm{p}}{ }^{2}=0.10\right]$.

The interaction analysis (Fig. 2) showed that functional activity in the subgenual and subcallosal ACC showed significant differences among conditions (i.e., increase> natural $>$ decrease) when there was less cognitive load for conflict monitoring (i.e., congruent) or error-preventive regulation was successful (i.e., subsequent correct). However, such a relationship was reversed under high cognitive conflict, and it was not significantly observed when the subsequent error was not prevented.

\section{Conjunction analyses}

We examined whether emotion regulation would show additive effects on action monitoring in the ACC. Even though we applied a single ACC ROI mask, we found some 
independent clusters for each action-monitoring condition in dorsal and rostral ACC.

Conflict monitoring (target congruency) No region in the ACC was significant for the conjunction analysis of Target Congruency $\times$ Scan and Emotion Regulation $\times$ Scan .

Error processing (current accuracy) There were two significant regions in the ACC for the conjunction analyses of Current Accuracy $\times$ Scan and Emotion Regulation $\times$ Scan $(p<$
$0.0025)$. One cluster, in the dACC (BA 24; centroid: $x=2, y=$ 10, $z=34$; see Fig. 3, top), showed higher activity after error commissions than after correct trials $(t=4.26, p<0.001, d=$ 1.51 ), and the time course of functional activity in the dACC cluster was significantly modulated by emotion regulation condition (i.e., decrease $<$ natural $<$ increase; linear trend $t=$ 2.37, $p<0.05, R^{2}=0.32$ ). In contrast, the other cluster, in rACC (BA 32; centroid: $x=12, y=44, z=6$; see Fig. 3, middle), showed lower activity after error commissions than after correct responses $(t=3.54, p<0.005, d=1.25)$; however,

Error Processing (current accuracy $\mathrm{x}$ scan and emotion regulation $\mathrm{x}$ scan)

Dorsal ACC [centroid: $\mathrm{x}=2, \mathrm{y}=10, \mathrm{z}=34$ ]
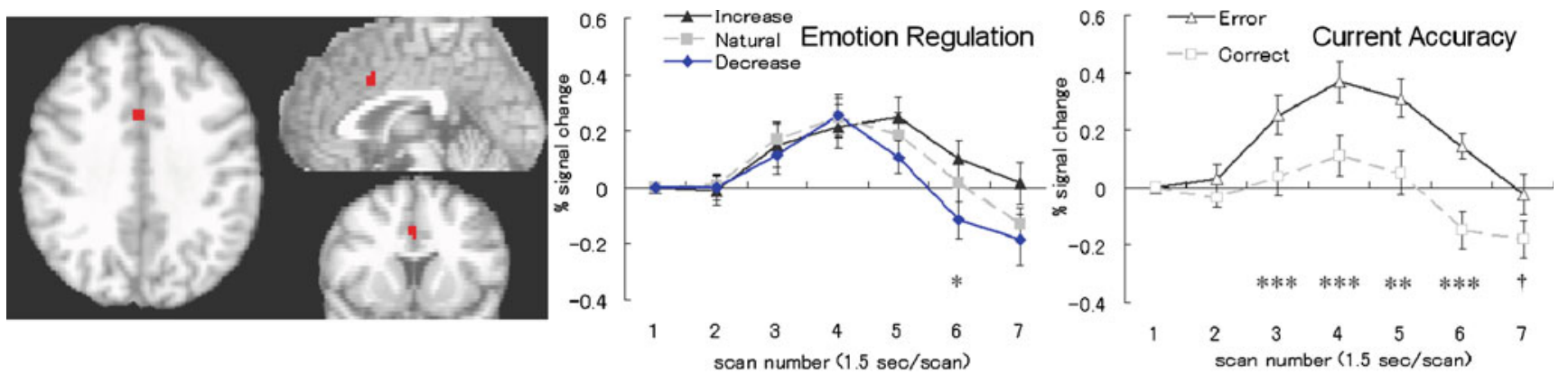

Rostral ACC [centroid: $\mathrm{x}=12, \mathrm{y}=44, \mathrm{z}=6$ ]
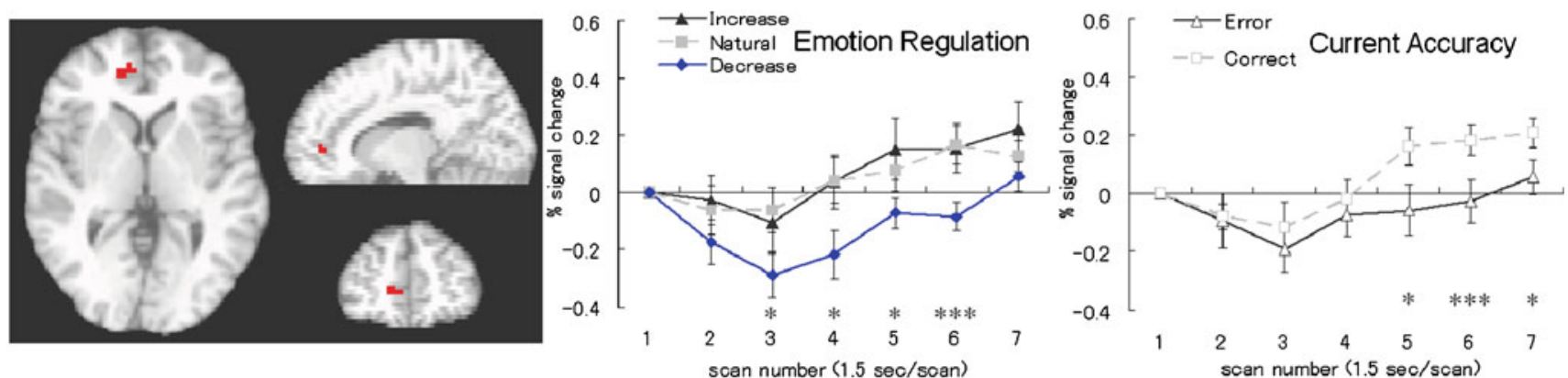

Error Prevention (subsequent accuracy x scan and emotion regulation x scan)

Rostral ACC [centroid: $\mathrm{x}=3, \mathrm{y}=41, \mathrm{z}=10$ ]
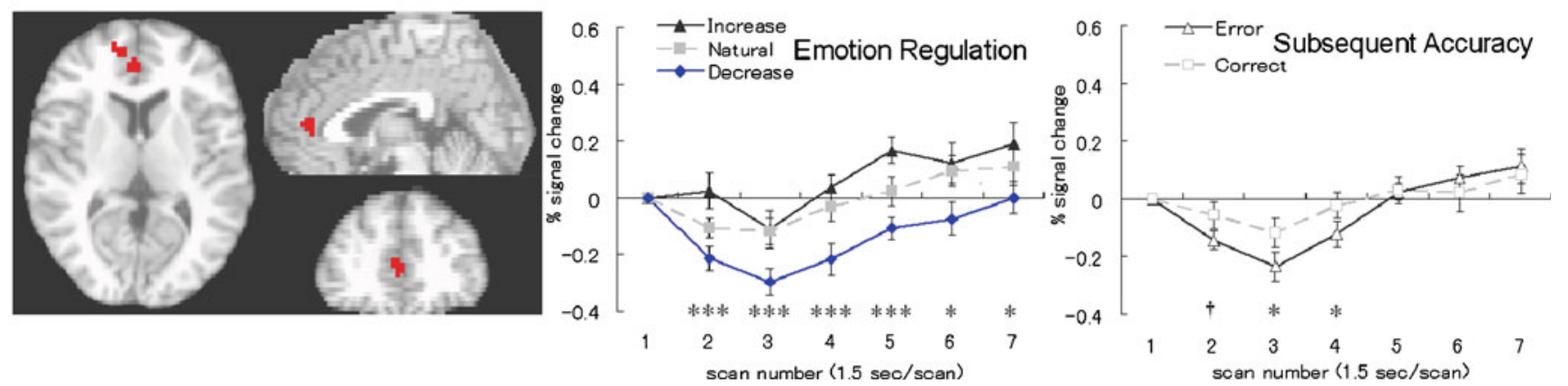

Fig. 3 Conjunction effects of emotion regulation and error processing/prevention in the ACC. Top, Middle: Dorsal and rostral ACC clusters and the time courses of their functional activity (error processing: Current Accuracy $\times$ Scan and Emotion Regulation $\times$ Scan) . Bottom: Rostral ACC cluster and its time course (error prevention: Subsequent Accuracy $\times$ Scan and Emotion Regulation $\times$ Scan) 

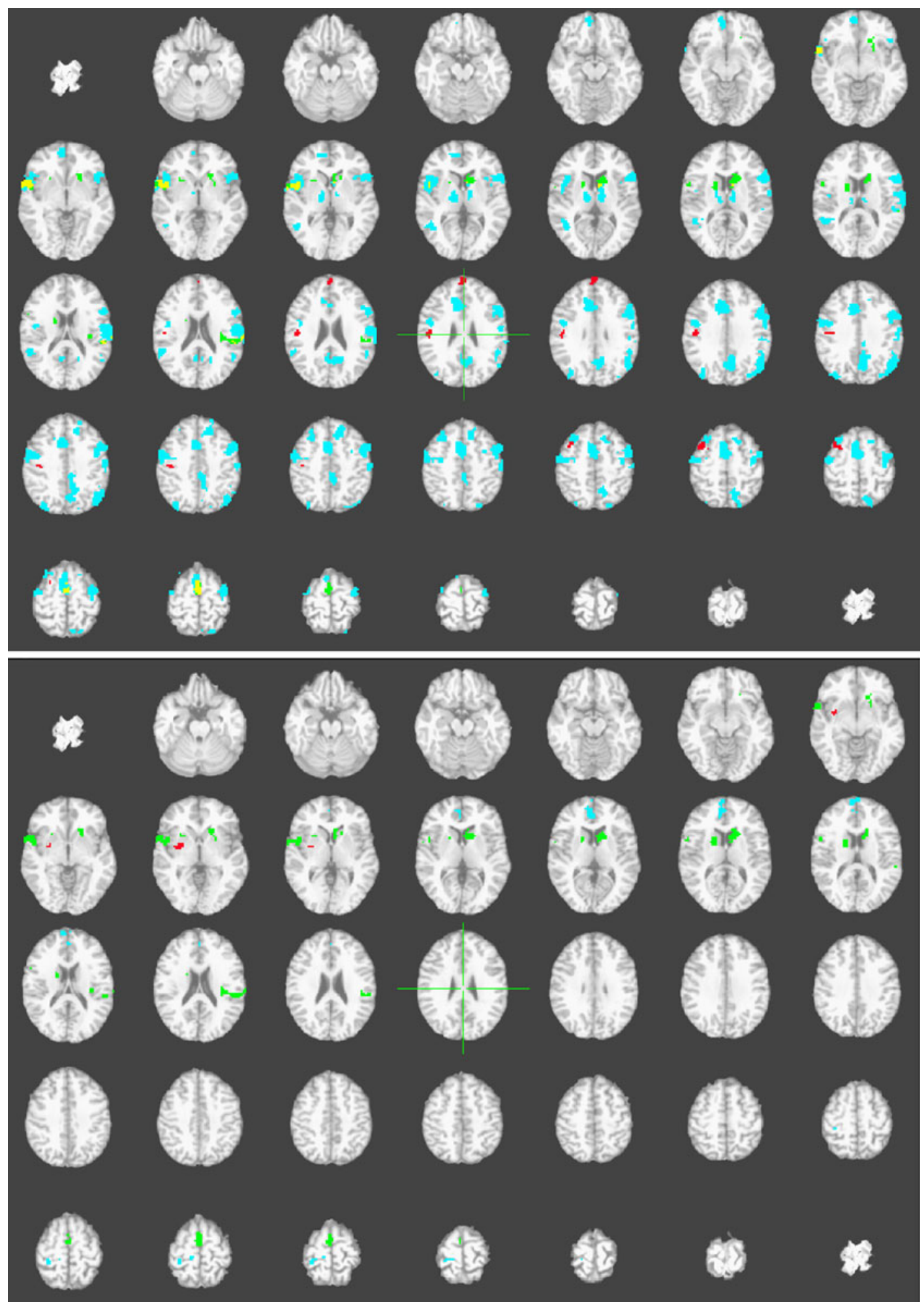

Fig. 4 Results of whole-brain analyses associated with error processing and prevention. (No region associated with conflict monitoring reached significance.) Top: Whole-brain analysis using current performance accuracy. Regions identified in each condition were restricted via empirically derived contiguity thresholding: Blue $=$ Current Accuracy $\times$ Scan interactions $(p<0.005)$; Green=Emotion Regulation $\times$ Scan interactions $(p<0.005)$; Red $=$ Emotion Regulation $\times$ Current Accuracy $\times$ Scan $(p<0.005)$; Yellow $=$ overlapping areas of the regions above. Bottom: Whole-brain analysis using subsequent performance accuracy. Regions identified in each condition were restricted via empirically derived contiguity thresholding: Blue $=$ Subsequent Accuracy $\times$ Scan interactions $(p<0.005$, subsequent error $<$ subsequent correct); Green $=$ Emotion Regulation $\times$ Scan interactions $(p<0.005$, decrease $<$ natural $<$ increase $)$; Red $=$ Emotion Regulation $\times$ Subsequent Accuracy $\times$ Scan interactions $(p<0.005)$. There were no overlapping areas of the regions above 
it showed the same pattern of activity as the dorsal cluster on emotion regulation effects at Scan 6 (i.e., decrease $<$ natural $<$ increase; linear trend $t=2.42, p<0.05, R^{2}=0.33$ ).

Error prevention (subsequent accuracy) A cluster in the rACC (BA 24; centroid: $x=3, y=41, z=10$; see Fig. 3, bottom) was significantly associated with both Subsequent Accuracy $\times$ Scan and Emotion Regulation $\times$ Scan. This region showed reduced activity in the decrease condition as compared to the natural and increase conditions at Scan 3 (i.e., decrease $<$ natural $<$ increase; linear trend $t=4.65, p<$ $0.001, R^{2}=0.57$ ). Decreased activity in this region was followed more often by subsequent performance errors than by correct responses $(t=2.44, p<0.05, d=0.89)$.

A conjunction analysis (Fig. 3) suggested that the dorsal ACC cluster (BA 24) was associated with both error processing (i.e., current accuracy) and emotion regulation independently, showing sustained activity around Scan 6 (i.e., around $9.0 \mathrm{~s}$ after the trial onset). The large rACC cluster (BA 24) was associated with both error prevention (i.e., subsequent accuracy) and emotion regulation, and the time course activity showed the most negative peak around Scan 3 (i.e., around $4.5 \mathrm{~s}$ after the trial onset). Interestingly, the smaller rACC cluster (BA 32) showed a pattern of activity that was similar to the patterns in both of those clusters. The most negative peak was around Scan 3, and the most significant differences among emotion regulation conditions were in the sustained activity around Scan 6 . Because anatomical localization of regions as dorsal or rostral cannot fully describe their functional differences (Etkin, Egner, \& Kalisch, 2011), we used a bivariate functional mixed-effects model, described below, to better understand the functional relationships between these regions.

\section{Whole-brain analyses}

To understand whether the observed ACC results were part of a broader network, Table 3 and Fig. 4 describe regions detected using voxel-wise whole-brain analyses associated with emotion regulation and each of conflict monitoring, error processing, and error prevention, using both conjunction and interaction analyses. Of particular note, errorprocessing analyses revealed an SMA region that was associated with both current accuracy and emotion regulation (conjunction analysis: $p<0.0025$; listed in Table 3). A cluster from dACC to SMA (BA 6) showed enhanced activity after error trials relative to after correct trials (Current Accuracy $\times$ Scan, $p<0.005$; see the blue-colored regions in Fig. 4, top), and the SMA region found in the whole-brain analysis was robustly associated with both emotion regulation and current accuracy, with a larger number of voxels than the dACC region, which showed the same pattern of activity as the ROI analysis. The SMA is often considered to be strongly associated with the dACC in function (e.g., Braver et al., 2001; Carter et al., 2000), and it has been associated with response selection (i.e., in forced choice tasks) and response inhibition (i.e., in go/ no-go tasks). The rACC (BA 24/32) region extending to the medial frontal gyrus (BA 10) was associated with both subsequent accuracy and emotion regulation. Thus, this SMA region was analyzed along with the $\mathrm{rACC} / \mathrm{MPFC}$ region in subsequent analyses.

Bivariate functional mixed effects model: Effective connectivity of error-processing and error-prevention regions

Our previous findings suggested that the rostral (rACC/ MPFC) and dorsal (SMA) regions are differentially modulated by emotion regulation instructions and action monitoring associated with task performance, such that there are stronger emotion-regulation-modulated involvements of dorsal regions in responding to current errors and of rostral regions in predicting subsequent errors. We hypothesized that these regions would not only each be affected, but that their relationships (i.e., synergistic functioning as a system) would be modulated by emotion regulation, and that this systemic functioning would lead to differential preparation for subsequent performance.

We tested this hypothesis using a bivariate functional mixed-effects model (Rosen \& Thompson, 2009) that simultaneously accounted for the time course of the regions of error processing and error prevention. We used the brain regions identified in the previous whole-brain analyses that had larger numbers of voxels and more robust differences among regulation conditions than the dACC region, which showed the same pattern of activity as the ROI analysis. Specifically, we examined the mutual dynamic influences of these regions over time by fitting a Bayesian bivariate functional mixed-effects model using WinBUGS Version 1.4.1 (Spiegelhalter, Thomas, Best, \& Lunn, 2003). The bivariate responses were BOLD activation (percent change from the first scan of a trial) in dorsal and rostral regions, with scans nested within trials nested within individuals.

The statistical model is given by

$$
\begin{gathered}
\mathbf{Y}_{i j}(t)=\mu(t)+\mathbf{g}_{i}(t)+\delta_{i j}(t)+\varepsilon_{i j}(t), \\
\delta_{i j}(t)=\Gamma \delta_{i j}(t-1)+\omega_{i j}(t), t=1, \ldots 7 .
\end{gathered}
$$

Here, the subscript $i$ denotes the individual, and the subscript $j$ denotes the trial nested within the individual. For the $(i, j)$ th trial at scan $t$, the activation within the dorsal and rostral regions is $\mathbf{Y}_{i j}(t)=\left[Y_{i j 1}(t), Y_{i j 2}(t)\right]$. The errors $\varepsilon_{i j}(t)=\left[\varepsilon_{i j 1}(t), \varepsilon_{i j 2}(t)\right]$ were modeled as independent, normal- 
ly distributed bivariate white noise processes. Overall mean levels of activation over scans in each region, denoted by $\boldsymbol{\mu}(t)=\left[\mu_{1}(t), \mu_{2}(t)\right]$, were modeled as a smooth linear combination of B-spline basis functions; individual variations from the mean activation curves, denoted by $\mathbf{g}_{i}(t)=$ $\left[g_{i 1}(t), g_{i 2}(t)\right]$, were modeled as normally distributed random coefficients on the same B-spline basis functions. The within-trial random effects $\delta_{i j}(t)$ were specified as a first-order bivariate autoregressive process, as can be seen in the second line of the model equation, with autoregressive matrix $\Gamma$ and independent multivariate normal innovations $\boldsymbol{\omega}_{i j}(t)$. The estimated off-diagonal coefficients of the $2 \times 2$ autoregression matrix $\Gamma$ relating lag- 1 scan residual BOLD activation $\mathbf{Y}_{i j}(t-1)$ to current scan BOLD activation $\mathbf{Y}_{i j}(t)$ were used to determine levels of effective connectivity between the two regions across the natural, decrease, and increase conditions. Vague Bayesian priors for this model were multivariate normal for the parameters in $\boldsymbol{\mu}(t), \mathbf{g}_{\mathrm{i}}(t)$, and $\Gamma$, inverse gamma for the dispersion parameters $\varepsilon_{\mathrm{ij}}(t)$, and inverse Wishart for the dispersion parameters $\omega_{\mathrm{ij}}(t)$.

The results of a Bayesian bivariate functional mixedeffects model using the activations of the error-processing region (SMA) and the error prevention region ( $\mathrm{rACC} /$ MPFC) from the conjunction analyses on current/subsequent accuracy and emotion regulation were as follows (Fig. 5). For the decrease condition, the connectivity coefficient for determining the past $\mathrm{rACC} / \mathrm{MPFC}$ region residual BOLD activation on current SMA region BOLD activation was $-0.178(95 \% \mathrm{CI}:-0.115,-0.056)$; for the

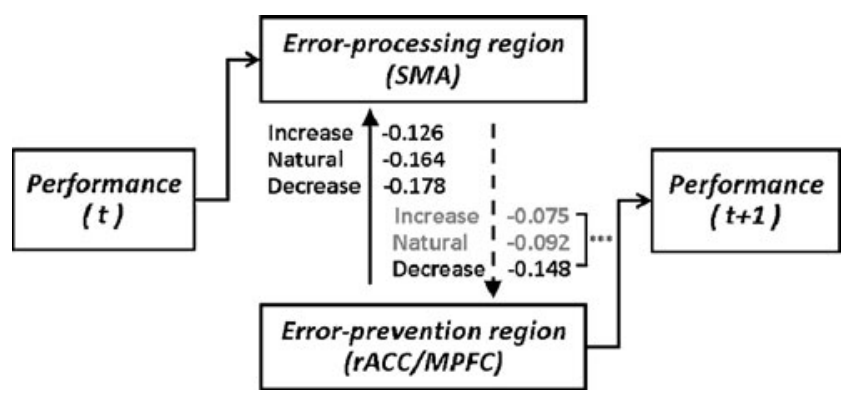

Fig. 5 Modeling results of the functional connectivity between the brain regions related to error processing and error prevention associated with task performance. The error-processing region (SMA) and the error prevention region ( $\mathrm{rACC}$ ) were defined from conjunction analyses $(p<0.0025)$ based on ANOVAs on response accuracy of the task [error or correct in current $(t)$ or subsequent $(t+1)$ trials; $p<0.05$ ] and on emotion regulation condition (natural, or decrease or increase emotional response; $p<0.05$ ). For functional connectivity between SMA and rACC, bivariate functional mixedeffects modeling was conducted separately on each emotion regulation condition. The rACC appeared to inhibit the SMA region in all of the conditions, but only in the decrease condition did the SMA inhibit the rACC. Solid lines=significant results in all of the emotion regulation conditions. Dashed line=significance depended on the condition (significant only in the decrease condition) natural condition, this coefficient was -0.164 (95\% CI: $0.119,-0.068)$; and for the increase condition, the coefficient was $-0.126(95 \%$ CI: $-0.064,-0.000)$. The corresponding coefficients for the effect of the past SMA region residual BOLD activation on current $\mathrm{rACC} / \mathrm{MPFC}$ BOLD activation were: decrease condition, -0.148 (95\% CI: $-0.106,-0.059)$; natural condition, -0.092 (95\% CI: $0.060,-0.032)$; and increase condition, -0.075 (95\% CI: $0.035,0.007)$. Causal relationships from the $\mathrm{rACC} / \mathrm{MPFC}$ region to the SMA region were significant in all the conditions, whereas that from the SMA to the rACC/MPFC region was significant only in the decrease condition. These results suggested that there were always significant inhibitory relationships from the region of error prevention (rACC/MPFC) to that of error processing (SMA) in any emotion regulation condition, whereas the opposite direction of inhibitory relationship from the region of error processing (SMA) to that of error prevention (rACC/ MPFC) was significant only when participants tried to decrease their emotional responses to errors.

\section{Discussion}

This study examined neural activity associated with explicit regulation of negative emotion caused by error commission, particularly with regard to functions of the action-monitoring system, including conflict monitoring, error processing, and error prevention. We asked participants to regulate their negative emotion associated with errors during a CPT by up- and down-regulation of their emotional state. A greater level of self-perceived negative emotion was associated with worse performance on the task in the natural condition, in contrast to the decrease and increase conditions, in which emotionality was not associated with performance, potentially because explicit emotion regulation modulated the levels of associations between negative emotion and task performance. This study had three principal findings: (1) sACC (BA 25) activity was modulated by emotion regulation under low cognitive conflict (congruent targets); the decrease condition was associated with decreased sACC activity, and the increase condition was associated with increased SACC activity. However, this association was not observed under high cognitive conflict (incongruent targets). This finding supports the idea that ventral action-monitoring regions are modulated by negative emotion, but emotional modulation is inhibited by an increased need for cognitive processing. (2) Dorsal and ventral action-monitoring regions showed the same pattern of modulation by emotion regulation, but a different pattern of activity associated with error processing. Both $\mathrm{dACC}$ and $\mathrm{rACC}$ activity showed the same pattern of modulation by emotion regulation (decrease $<$ 
natural $<$ increase); however, dACC showed increased activity after errors (error $>$ correct), whereas rACC showed decreased activity after errors (error $<$ correct). (3) Inhibitory relationships were significant between dorsal and ventral regions associated with both emotion regulation and error processing (SMA) or error prevention (rACC/MPFC) on a trial-by-trial basis. That is, when one explicitly tries to decrease negative emotion to errors, if there is earlier rACC/MPFC activity that is associated with error prevention and regulation, there would be less sustained SMA activity, which is associated with error processing and motor response. Then, if there is less sustained SMA activity, there would be more $\mathrm{rACC} / \mathrm{MPFC}$ regulatory activity in the subsequent trial. Importantly, we found that the functional activity of $\mathrm{rACC} / \mathrm{MPFC}$ is associated with decreased activity in the SMA in all of the emotion regulation conditions, possibly reflecting inhibition. In contrast, SMA is associated with decreased $\mathrm{rACC} / \mathrm{MPFC}$ activity only in the decrease condition, during which participants were asked to decrease their negative emotion to errors by explicit emotion regulation instructions.

Though neural mechanisms of instructed regulation of error-related emotional reactions could have been different from those identified in studies in which negative emotion was regulated using passive viewing of affective pictures, our data suggest striking similarities to that literature. Our manipulation of error-related emotion regulation modulated $\mathrm{rACC} / \mathrm{sACC}$ activity, which was associated in previous studies with self-related negative emotion and affective response conflict with emotional interference. For example, $\mathrm{rACC} / \mathrm{sACC}$ is associated with a negative mood state (George, Ketter, Parekh, Horwitz, Herscovitch, \& Post, 1995), emotion regulation of negative autobiographical memories (Kross, Davidson, Weber, \& Ochsner, 2009), processing of self-related negative words (Yoshimura, Ueda, Suzuki, Onoda, Okamoto, \& Yamawaki, 2009), severity of depressive symptoms (Yoshimura, Okamoto, Onoda, Matsunaga, Ueda, Suzuki et al., 2010), autonomic arousal level (in a Stroop task, Critchley, Tang, Glaser, Butterworth, \& Dolan, 2005; in a two-alternative forcedchoice task, Ohira et al., 2010), and a potentially frustrating cognitive task in vulnerable populations (antisocial personality disorder; Völlm et al., 2010). However, we have thus added to this literature the idea that $\mathrm{rACC} / \mathrm{sACC}$ is modulated by explicitly regulated negative emotion after errors, and such modulation pattern could be changed in conditions where more cognitive activity is required or where a likelihood of error commission is high.

Another primary contribution of this study to the literature is that we required affect regulation to errors, rather than using traditional emotion regulation tasks that have used explicitly affective stimuli to evoke emotional responses. For example, in previous studies, externally evoked phasic emotional responses were observed using affective pictures, whereas internally induced tonic emotional responses, including anticipatory anxiety, were examined using loud tones or speech preparations (see, e.g., Davidson, 2002, for a review). However, in this study, participants generated phasic emotional reactions to their error responses in a cognitive task. We presented neither affective stimuli nor performance feedback during the experiment. In addition, participants could not predict when they would dynamically make errors during the task, and thus could not explicitly prepare for errors, which is different from viewing pictures, which could be prepared for during fixations or presentation of cue stimuli. That said, as our results are quite consistent with the broader emotion regulation literature, we can suggest that features of emotional reactivity and regulation of errors may be similar to reactivity and regulation of other types of emotional information.

Our results also suggest that activity in functional ACC subdivisions was modulated in the same way by regulation of negative emotion but differently by error processing. One might have guessed that rACC activity should have increased after errors, due to negative emotion. But instead, the reverse pattern was observed between dorsal and rostral regions, with effective connectivity analyses also supporting an inhibitory relationship (Fig. 5). That is, though the rACC generally showed overall increased activity in the increase and natural conditions (Fig. 3, top and middle right), those regions showed reverse patterns associated with task performance (Fig. 3, top and middle left). There was no interaction effect observed for error processing (but there was for other conflict processing and error prevention in ACC; Fig. 2). Though two of the rostral clusters were observed in conjunction analyses, the larger rostral cluster associated with error prevention showed significant differences in early scans, whereas the dorsal and smaller rostral clusters, which were associated with error processing, showed significant differences in later scans. These results support the idea that these ACC subdivisions do not serve redundant functionality.

Our observation that explicit emotion regulation modulates a dorsal action-monitoring region (SMA) and a ventral action-monitoring region ( $\mathrm{rACC} / \mathrm{MPFC}$ ) differently is consistent with the previous literature. For example, cognitive tasks that use emotional stimuli demonstrate inhibitory associations between $\mathrm{rACC}$ and dACC/SMA (e.g., Bush et al., 2000; Williams et al., 2006). In this study, conscious regulation of negative emotion after errors modulated inhibitory relationships between rostral and dorsal action-monitoring regions; the inhibitory relationship from $\mathrm{rACC}$ /MPFC to SMA was significant regardless of the emotion regulation condition, whereas the inhibitory relationship from SMA to $\mathrm{rACC} / \mathrm{MPFC}$ was significant only in the decrease condition. These data suggested that higher 
rostrally modulated emotional reactivity causes lower dorsally modulated error-processing, regardless of explicit regulation. The dorsal system only appears to exert regulatory control over the ventral system in the presence of an explicit intention to decrease emotional reactions.

Clusters in the right medial frontal gyrus, bilateral insula, right superior temporal gyrus, left striatum (caudate, putamen), right precentral gyrus, and left postcentral gyrus showed greater sustained activity in the increase condition than in the other two conditions (i.e., Emotion Regulation $\times$ Scan interaction, $p<0.005$; green-colored regions in Fig. 4, top). This pattern is consistent with previous emotion regulation research using a passive viewing task (e.g., Ochsner et al., 2004). Though the previous studies found that those regions were activated in both decrease and increase conditions with reappraisal, as compared to a natural ("look") condition, there was no error-related region that showed greater activation in the decrease condition than in the natural condition in this study. This could be because "error-related" negative emotion is naturally more associated with self-related processing than is passive viewing of emotional pictures. In this case, self-processing regions would be activated for self-control in proportion to negative emotion caused by errors.

In order to check the strategies actually used to regulate error-related emotions by each individual, we coded free descriptions reported by participants after the experiment. The results showed that participants used cognitive reappraisal mostly in the decrease condition (i.e., positive reappraisal) and also in the increase condition (i.e., negative reappraisal), as compared to the suppression to reduce emotion-expressive behavior (see details of the coding procedure and results in Table S1 and Fig. S3 in the supplementary materials).

There are several limitations to the present investigation. The sample was small $(n=17)$; larger sample sizes are increasingly standard. Thus, some of our effects that were not significant but were nearly so (e.g., effects of sACC on subsequent accuracy $[p=0.11]$ may have been significant with a larger sample). That said, since our sample was balanced on gender $(\sim 50 / 50)$, it is likely that we did not have systematic gender biases in our results. In this study, we focused on functions that are common, regardless of gender. That said, future studies may benefit from examining gender differences, since they have been observed in emotional reactivity to pictures in the amygdala and striatum (McRae, Ochsner, Mauss, Gabrieli, \& Gross, 2008). In a debriefing session, participants reported that they used cognitive reappraisal more than suppression as a regulation strategy (see Table S1 and Fig. S3 in the supplementary materials), but they may have used several different strategies to regulate their emotional responses to errors. This could have induced significant variability in neural activity. Error commission could naturally cause negative emotional responses high enough that the subjective ratings on emotional arousal were not significantly different between the increase and natural conditions. This could be partially due to task order effects, because the half of the participants who completed the decrease condition before the increase condition could have retained the strong sentiment of the decrease instructions (e.g., "focus on how poorly this reflects on your intelligence") into the subsequent blocks. In support of this idea, responses were significantly slower after errors in blocks following the decrease instruction. Future research using between-groups designs could get around this limitation. Though participants reported that they tried to have natural emotional reactions, they tended to focus more on the cognitive task in order to be less conscious and to have less control on their emotional reactions (Table S1 and Fig. S3 in the supplementary materials). Though RTs were faster on error trials than on correct trials in all the emotion regulation conditions, error-related ACC activity was still present when RT was used as a covariate (Fig. S4 in the supplementary materials). We applied a mixed-event design that included both rapid trials (430 to $\sim 640 \mathrm{~ms}$ per trial) and slow trials (an additional 10-s blank period following the stimulus). This manipulation might have had unanticipated effects, though no participants reported that they noticed the different frequencies of slow trials after correct and error responses (i.e., in order to get almost the same number of slow events for correct and error responses). This design allowed us to collect more error trials for analysis, but the standard deviation of the number of error trials was still comparatively high.

In summary, we examined the effects of explicit emotion regulation on brain activity associated with action monitoring. The results suggested that both action monitoring and explicit emotion regulation modulate functional activity throughout the ACC in response to cognitive conflict, error processing, and preparation for the subsequent trial. Further, this modulation appears to affect communication between rostral and dorsal action-monitoring systems. Together, these data suggest that even "cognitive" tasks on which errors occur may be subject to neural activity associated with both action system functions and an individual's own tendencies and strategies for regulating their emotions in dorsal and ventral regions. In the presence of explicit emotion regulation instructions, this modulation can be clearly observed. These dual influences of emotional and cognitive processes may be particularly important in populations in which explicit difficulties with emotion regulation are observed, such as clinical depression and anxiety.

Author Note We thank Monica Barback for comments on the experiment design, Kyung Hwa Lee for comments on the fMRI analyses, Agnes Haggerty for assistance of collecting data, and many members of the Program in Cognitive Affective Neuroscience lab for 
their comments, suggestions, and proofreading on previous versions of this manuscript. This work was supported by the Japanese Society for Promotion of Science (JSPS), the Pittsburgh MR Center Pilot Neuroimaging Program, and MH064159.

\section{References}

Barch, D. M., Braver, T. S., Akbudak, E., Conturo, T., Ollinger, J., \& Snyder, A. (2001). Anterior cingulate cortex and response conflict: Effects of response modality and processing domain. Cerebral Cortex, 11, 837-848.

Beauregard, M., Lévesque, J., \& Bourgouin, P. (2001). Neural correlates of conscious self-regulation of emotion. Journal of Neuroscience, 21, RC165.

Botvinick, M. M. (2007). Conflict monitoring and decision making: Reconciling two perspectives on anterior cingulate function. Cognitive, Affective, \& Behavioral Neuroscience, 7, 356-366.

Botvinick, M. M., Cohen, J. D., \& Carter, C. S. (2004). Conflict monitoring and anterior cingulate cortex: An update. Trends in Cognitive Sciences, 8, 539-546.

Botvinick, M., Nystrom, L. E., Fissell, K., Carter, C. S., \& Cohen, J. D. (1999). Conflict monitoring versus selection-for-action in anterior cingulate cortex. Nature, 402, 179-181.

Braver, T. S., Barch, D. M., Gray, J. R., Molfese, D. L., \& Snyder, A. (2001). Anterior cingulate cortex and response conflict: Effects of frequency, inhibition and errors. Cerebral Cortex, 11, 825836.

Bush, G., Luu, P., \& Posner, M. I. (2000). Cognitive and emotional influences in anterior cingulate cortex. Trends in Cognitive Sciences, 4, 215-222.

Bush, G., Shin, L. M., Holmes, J., Rosen, B. R., \& Vogt, B. A. (2003). The multi-source interference task: Validation study with fMRI in individual subjects. Molecular Psychiatry, 8, 60-70.

Carter, C. S., Braver, T. S., Barch, D. M., Botvinick, M. M., Noll, D., \& Cohen, J. D. (1998). Anterior cingulate cortex, error detection, and the online monitoring of performance. Science, 280, 747-749.

Carter, C. S., Macdonald, A. M., Botvinick, M., Ross, L. L., Stenger, V. A., Noll, D., et al. (2000). Parsing executive processes: strategic vs. evaluative functions of the anterior cingulate cortex. Proceedings of the National Academy of Sciences, 97, 1944-1948.

Cavanagh, J. F., Gründler, T. O., Frank, M. J., \& Allen, J. J. (2010). Altered cingulate sub-region activation accounts for task-related dissociation in ERN amplitude as a function of obsessivecompulsive symptoms. Neuropsychologia, 48, 2098-2109.

Cox, R. W. (1996). AFNI: Software for analysis and visualization of functional: Magnetic resonance neuroimages. Computers and Biomedical Research, 29, 162-173.

Critchley, H. D., Tang, J., Glaser, D., Butterworth, B., \& Dolan, R. J. (2005). Anterior cingulate activity during error and autonomic response. NeuroImage, 27, 885-895.

Davidson, R. J. (2002). Anxiety and affective style: Role of prefrontal cortex and amygdala. Biological Psychiatry, 51, 68-80.

Davidson, R. J., Lewis, D. A., Alloy, L. B., Amaral, D. G., Bush, G., Cohen, J. D., et al. (2002). Neural and behavioral substrates of mood and mood regulation. Biological Psychiatry, 52, 478-502.

Davis, K. D., Hutchison, W. D., Lozano, A. M., Tasker, R. R., \& Dostrovsky, J. O. (2000). Human anterior cingulate cortex neurons modulated by attention-demanding tasks. Journal of Neurophysiology, 83, 3575-3577.

Davis, K. D., Taylor, K. S., Hutchison, W. D., Dostrovsky, J. O., McAndrews, M. P., Richter, E. O., et al. (2005). Human anterior cingulate cortex neurons encode cognitive and emotional demands. Journal of Neuroscience, 25, 8402-8406.
Egner, T., Etkin, A., Gale, S., \& Hirsch, J. (2008). Dissociable neural systems resolve conflict from emotional versus nonemotional distracters. Cerebral Cortex, 18, 1475-1484.

Engels, A. S., Heller, W., Spielberg, J. M., Warren, S. L., Sutton, B. P., Banich, M. T., et al. (2010). Co-occurring anxiety influences patterns of brain activity in depression. Cognitive, Affective, \& Behavioral Neuroscience, 10, 141-156.

Etkin, A., Egner, T., \& Kalisch, R. (2011). Emotional processing in anterior cingulate and medial prefrontal cortex. Trends in Cognitive Sciences, 15, 85-93.

Etkin, A., Egner, T., Peraza, D. M., Kandel, E. R., \& Hirsch, J. (2006). Resolving emotional conflict: a role for the rostral anterior cingulate cortex in modulating activity in the amygdala. Neuron, $51,871-882$.

Falkenstein, M., Hoormann, J., Christ, S., \& Hohnsbein, J. (2000). ERP components on reaction errors and their functional significance: a tutorial. Biological Psychology, 51, 87-107.

First, M. B., Spitzer, R. L., Gibbon, M., \& Williams, J. B. W. (1996). Structured clinical interview for DSM-IV Axis I disordersPatient edition. New York: Biometrics Research Department, New York State Psychiatric Institute.

Fissell, K., Tseytlin, E., Cunningham, D., Iyer, K., Carter, C. S., Schneider, W., et al. (2003). Fiswidgets: A graphical computing environment for neuroimaging analysis. Neuroinformatics, 1, 111-125.

Gehring, W. J., Himle, J., \& Nisenson, L. G. (2000). Action-monitoring dysfunction in obsessive-compulsive disorder. Psychological Science, 11, 1-6.

George, M. S., Ketter, T. A., Parekh, P. I., Horwitz, B., Herscovitch, P., \& Post, R. M. (1995). Brain activity during transient sadness and happiness in healthy women. American Journal of Psychiatry, 152, 341-351.

Gross, J. J. (1998). Antecedent- and response-focused emotion regulation: Divergent consequences for experience, expression, and physiology. Journal of Personality and Social Psychology, 74, 224-237.

Holmes, A. J., \& Pizzagalli, D. A. (2008). Spatiotemporal dynamics of error processing dysfunctions in major depressive disorder. Archives of General Psychiatry, 65, 179-188.

Johnson, M. K., Nolen-Hoeksema, S., Mitchell, K. J., \& Levin, Y. (2009). Medial cortex activity, self-reflection and depression. Social Cognitive and Affective Neuroscience, 4, 313-327.

Kross, E., Davidson, M., Weber, J., \& Ochsner, K. (2009). Coping with emotions past: the neural bases of regulating affect associated with negative autobiographical memories. Biological Psychiatry, 65, 361-366.

Luu, P., Tucker, D. M., Derryberry, D., Reed, M., \& Poulsen, C. (2003). Electrophysiological responses to errors and feedback in the process of action regulation. Psychological Science, 14, $47-53$.

Mathalon, D. H., Whitfield, S. L., \& Ford, J. M. (2003). Anatomy of an error: ERP and fMRI. Biological Psychology, 64, 119-141.

McCormick, L. M., Ziebell, S., Nopoulos, P., Cassell, M., Andreasen, N. C., \& Brumm, M. (2006). Anterior cingulate cortex: An MRIbased parcellation method. NeuroImage, 32, 1167-1175.

McRae, K., Ochsner, K. N., Mauss, I. B., Gabrieli, J. D. E., \& Gross, J. J. (2008). Gender differences in emotion regulation: An fMRI study of cognitive reappraisal. Group Processes \& Intergroup Relations, 11, 143-162.

Mohanty, A., Engels, A. S., Herrington, J. D., Heller, W., Ho, M. H., Banich, M. T., et al. (2007). Differential engagement of anterior cingulate cortex subdivisions for cognitive and emotional function. Psychophysiology, 44, 343-351.

Nieuwenhuis, S., Holroyd, C. B., Mol, N., \& Coles, M. G. (2004). Reinforcement-related brain potentials from medial frontal cortex: Origins and functional significance. Neuroscience and Biobehavioral Reviews, 28, 441-448. 
Ochsner, K. N., Hughes, B., Robertson, E. R., Cooper, J. C., \& Gabrieli, J. D. E. (2009). Neural systems supporting the control of affective and cognitive conflicts. Journal of Cognitive Neuroscience, 21, 1842-1855.

Ochsner, K. N., Ray, R. D., Cooper, J. C., Robertson, E. R., Chopra, S., Gabrieli, J. D. E., et al. (2004). For better or for worse: Neural systems supporting the cognitive down- and up-regulation of negative emotion. NeuroImage, 23, 483-499.

Ohira, H., Ichikawa, N., Nomura, M., Isowa, T., Kimura, K., Kanayama, N., et al. (2010). Brain and autonomic association accompanying stochastic decision-making. NeuroImage, 49, 1024-1037.

Pellegrino, G., Ciaramelli, E., \& Làdavas, E. (2007). The regulation of cognitive control following rostral anterior cingulate cortex lesion in humans. Journal of Cognitive Neuroscience, 19, 275-286.

Ray, R. D., Ochsner, K. N., Cooper, J. C., Robertson, E. R., Gabrieli, J. D. E., \& Gross, J. J. (2005). Individual differences in trait rumination and the neural systems supporting cognitive reappraisal. Cognitive, Affective, \& Behavioral Neuroscience, 5, 156-168.

Rosen, O., \& Thompson, W. (2009). A Bayesian regression model for multivariate functional data. Journal of Computational Statistics and Data Analysis, 53, 3773-3786.

Spiegelhalter, D., Thomas, A., Best, N., \& Lunn, D. (2003). WinBUGS user manual, version 1.4. Cambridge: Medical Research Council Biostatistics Unit.

Talairach, J., \& Tournoux, P. (1988). Co-planar stereotaxic atlas of the human brain: 3-dimensional proportional system. An approach to cerebral imaging. New York: Thieme Medical.

Ullsperger, M., \& von Cramon, D. Y. (2001). Subprocesses of performance monitoring: A dissociation of error processing and response competition revealed by event-related fMRI and ERPs. NeuroImage, 14, 1387-1401.
Ursu, S., Stenger, V. A., Shear, M. K., Jones, M. R., \& Carter, C. S. (2003). Overactive action monitoring in obsessive-compulsive disorder: evidence from functional magnetic resonance imaging. Psychological Science, 14, 347-353.

van Veen, V., \& Carter, C. S. (2002). The timing of action-monitoring processes in the anterior cingulate cortex. Journal of Cognitive Neuroscience, 14, 593-602.

van Veen, V., Cohen, J. D., Botvinick, M. M., Stenger, V. A., \& Carter, C. S. (2001). Anterior cingulate cortex, conflict monitoring, and levels of processing. Neurolmage, 14, 1302-1308.

Völlm, B., Richardson, P., McKie, S., Reniers, R., Elliott, R., Anderson, I. M., et al. (2010). Neuronal correlates and serotonergic modulation of behavioural inhibition and reward in healthy and antisocial individuals. Journal of Psychiatric Research, 44, 123-131.

Williams, L. M., Kemp, A. H., Felmingham, K., Barton, M., Olivieri, G., Peduto, A., et al. (2006). Trauma modulates amygdala and medial prefrontal responses to consciously attended fear. NeuroImage, 29, 347-357.

Woods, R. P., Cherry, S. R., \& Mazziotta, J. C. (1992). Rapid automated algorithm for aligning and reslicing PET images. Journal of Computer Assisted Tomography, 16, 620-633.

Yoshimura, S., Okamoto, Y., Onoda, K., Matsunaga, M., Ueda, K., Suzuki, S., et al. (2010). Rostral anterior cingulate cortex activity mediates the relationship between the depressive symptoms and the medial prefrontal cortex activity. Journal of Affective Disorders, 122, 76-85.

Yoshimura, S., Ueda, K., Suzuki, S., Onoda, K., Okamoto, Y., \& Yamawaki, S. (2009). Self-referential processing of negative stimuli within the ventral anterior cingulate gyrus and right amygdala. Brain and Cognition, 69, 218-225. 